\title{
Fibroblast Growth Factor 21 Predicts and Promotes Vascular Calcification in Haemodialysis Patients
}

\author{
Liqiong Jiang ${ }^{\mathrm{a}, \mathrm{b}}$ Qing Yin ${ }^{\mathrm{a}}$ Min Yang ${ }^{\mathrm{c}}{\text { Min } \mathrm{Li}^{\mathrm{c}} \text { Mingming Pan }}^{\mathrm{a}}$ \\ Yuchen Han ${ }^{\mathrm{a}}$ Zhen Zhao $^{\mathrm{d}}$ Zhi Wang $^{\mathrm{d}}$ Lili Zhu $^{\mathrm{a}}$ Qing Wei $^{\mathrm{a}}$ Yan Tu $^{\mathrm{a}}$ \\ Min Gao ${ }^{a}$ Hong Liu $^{a}$ Xiaoliang Zhanga Bi-cheng Liu ${ }^{a}$ Bin Wang ${ }^{a}$
}

anstitute of Nephrology, Zhong Da Hospital, Southeast University School of Medicine, Nanjing, China; ${ }^{b}$ Department of Nephrology, The Affiliated Suzhou Hospital of Nanjing Medical University, Suzhou, China; ' ${ }^{\text {Department of }}$

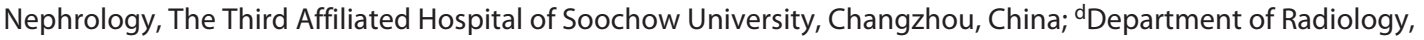
Zhong Da Hospital, Southeast University School of Medicine, Nanjing, China

\section{Keywords}

Fibroblast growth factor 21 - Vascular calcification .

Haemodialysis · Endothelial-to-mesenchymal transition

\begin{abstract}
Background: Cardiovascular disease (CVD) is the leading cause of death in haemodialysis (HD) patients. Vascular calcification (VC) is dramatically accelerated and is strongly associated with CVD events and mortality in HD patients. VC coexists with osteoporosis in many studies. Fibroblast growth factor 21 (FGF21) which is known as an adipocytokine is a new hypoglycemic strategy and is inversely related to bone mineral density. Methods: To evaluate the contribution of FGF21 to VC in HD patients, we detected circulating FGF21 levels and measured the whole thoracic aorta calcification scores (TACS) and calcification scores of the $3 \mathrm{seg}$ ments of thoracic aorta, including ascending thoracic aorta (ATACS), aortic arch (AoACS), and descending thoracic aorta (DTACS) of our HD patients in this cross-sectional study. In addition, we pre-incubated human aortic endothelial cells (HAECs) with FGF21 in the presence or absence of parathyroid hormone (PTH) in vitro. Results: The median serum
\end{abstract}

karger@karger.com www.karger.com/kdd

Karger $\stackrel{\text { ' }}{5}$

GOPEN ACCESS
(C) 2021 The Author(s)

Published by S. Karger AG, Basel

This is an Open Access article licensed under the Creative Commons Attribution-NonCommercial-4.0 International License (CC BY-NC) (http://www.karger.com/Services/OpenAccessLicense), applicable to the online version of the article only. Usage and distribution for commercial purposes requires written permission.
FGF21 level in HD patients was 11-fold higher than that in healthy controls. $\operatorname{Ln}(\mathrm{FGF} 21)$ was positively correlated with $\operatorname{Ln}(T A C S+1), \operatorname{Ln}($ ATACS +1), Ln(AoACS +1), and $\operatorname{Ln}($ DTACS +1), respectively, in HD patients. Serum FGF21 was independently associated with TACS and ATACS, AoACS, and DTACS. FGF21 which was combined with age, calcium, and intact PTH demonstrated a high area under the curve of 0.84 with optimal sensitivity (84\%) and specificity (71\%) for the prediction of VC in HD patients. Our vitro results showed that FGF21 enhanced the calcification effect of PTH on HAECs by increasing calcium deposition and endothelial-to-mesenchymal transition. Conclusions: Circulating FGF21 was notably higher and was a potential predictor and promoter of VC in HD patients.

(c) 2021 The Author(s) Published by S. Karger AG, Basel

\section{Introduction}

Mortality rates for patients with end-stage renal disease (ESRD) who were treated with dialysis remain unacceptably high, and the annual mortality rates range from 15 to $20 \%$ [1]. Cardiovascular disease (CVD) is the lead- 
ing cause of death among haemodialysis (HD) patients [2]. Many evidences indicate that abnormalities of bone and mineral metabolism in chronic kidney disease (CKD) may contribute to the development of CVD and increased cardiovascular mortality, with the most likely link being the development of vascular calcification (VC) $[3,4]$. VC progresses rapidly in $\mathrm{HD}$ patients, and the occurrence of $\mathrm{VC}$ among $\mathrm{HD}$ patients ranges from 70 to $90 \%$ [5-7]. VC is thought to be a powerful independent risk factor for CVD events and mortality in HD patients [8, 9]. A number of molecules involved in bone and mineral metabolism may also play a key role in cardiovascular calcification formation [9].

The human fibroblast growth factor (FGF) family includes 22 members [10]. FGF23 and FGF21 are endocrine factors that structurally belong to the FGF19 subfamily, which are released into circulation and exert systemic action [11]. Serum FGF23 levels increase up to $100-1,000$-folds in HD patients [12-14]. The involvement of elevated FGF23 in the progression of VC is controversial. Clinical studies indicate a correlation between higher FGF23 levels and increased aortic calcification [15, 16]. However, other studies have shown that FGF23 had no impact on VC $[17,18]$ or inhibited the progression of VC $[19,20]$.

FGF21 is also increased progressively with a decline of renal function $[21,22]$. Circulating FGF21 is mainly secreted from the liver as a metabolic regulator of glucose metabolism and lipid metabolism in response to fasting [23]. In obese rodents, it causes weight loss [24] and improves insulin sensitivity $[25,26]$. In humans, circulating FGF21 is inversely related to bone mineral density [27]. Elevated FGF21 levels are associated with reduced bone strength and with bone mass [28]. FGF21 analogues also increased blood markers of bone loss in 2 of the human studies $[29,30]$. Many studies have demonstrated an independent association between osteoporosis and VC [31, 32]. The role of FGF 21 in VC has rarely been reported. Therefore, whether circulating FGF21 is associated with $\mathrm{VC}$ is worth further investigation.

Endothelial cells (ECs) can undergo a process known as endothelial-to-mesenchymal transition (EndMT), which involves the loss of endothelial features and the acquisition of a fibroblast-like phenotype, eventually leading to cells with osteogenic potential [33]. In EndMT, ECs lose their endothelial markers, for example, platelet endothelial cell adhesion molecule (PECAM-1/CD31), von Willebrand factor, and vascular endothelial cadherin (VE-cadherin), and they acquire mesenchymal markers, for example, $\alpha$-smooth muscle actin ( $\alpha$-SMA), fibroblast special protein (FSP1), and vimentin [34]. However, the role of FGF21 in EndMT and calcium deposition in ECs under uraemic stress has not been reported.

This cross-sectional study aimed to achieve 4 objectives. The first objective was to quantify the correlation between FGF21 and VC. The second objective was to determine whether FGF21 is a novel risk factor for VC independent of traditional risk factors. The third objective was to examine the predictive effect of FGF21 on VC. The fourth objective was to identify the role and explore the underlying mechanism of FGF21 in the process of VC, especially focussing on the EndMT process, in cultured ECs.

\section{Materials and Methods}

\section{Study Design and Population}

This cross-sectional study included $802 \mathrm{HD}$ patients from 2 large HD centres, Nanjing Zhongda Hospital $(n=450)$ and the First People's Hospital of Changzhou $(n=352)$, China, from January 2018 to December 2018. The exclusion criteria are described in the flowchart shown in Figure 1. Finally, $388 \mathrm{HD}$ patients with chest multi-slice computed tomography (MSCT) examination within half a year and consenting to give blood samples were invited to this study. The enroled patients underwent stable regular $\mathrm{HD}$ using bicarbonate dialysate. Most patients received $4 \mathrm{~h}$ of $\mathrm{HD}$ treatment every session 3 times each week. In addition, we obtained data from 20 healthy controls from the physical examination centre of Nanjing Zhongda Hospital.

\section{Clinical and Biochemical Data Collection}

Details of collection of clinical and biochemical data are provided in the online suppl. material; for all online suppl. material, see www.karger.com/doi/10.1159/000512750.

\section{Measurement of Serum FGF21 and FGF23 in HD Patients}

Serum FGF21 levels were assessed by using enzyme-linked immunosorbent assay (ELISA) kits for human FGF21 (Neobioscience, China). Serum FGF23 levels were assessed by using ELISA kits for human FGF23 (Joyee Biotechnics, China). The levels of serum FGF21 and FGF23 were measured according to the manufacturers' instructions. Details of the measurements are provided in the online suppl. material.

\section{Assessment of Vascular Calcification}

The measurements and definitions of thoracic aorta calcification score (TACS), ascending thoracic aorta calcification score (ATACS), aortic arch calcification score (AoACS), and descending thoracic aorta calcification score (DTACS) in this study are provided in the online suppl. material.

\section{Definition of Light VC and Moderate/Severe VC}

The calcification scores of all segments were measured. First, HD patients were divided into 2 groups according to the median TACS $\left(0.77 \mathrm{~cm}^{3}\right)$ : the low TACS group $\left(<0.77 \mathrm{~cm}^{3}\right)$ and the high TACS group $\left(\geq 0.77 \mathrm{~cm}^{3}\right)$. The extents of TAC in HD patients were 


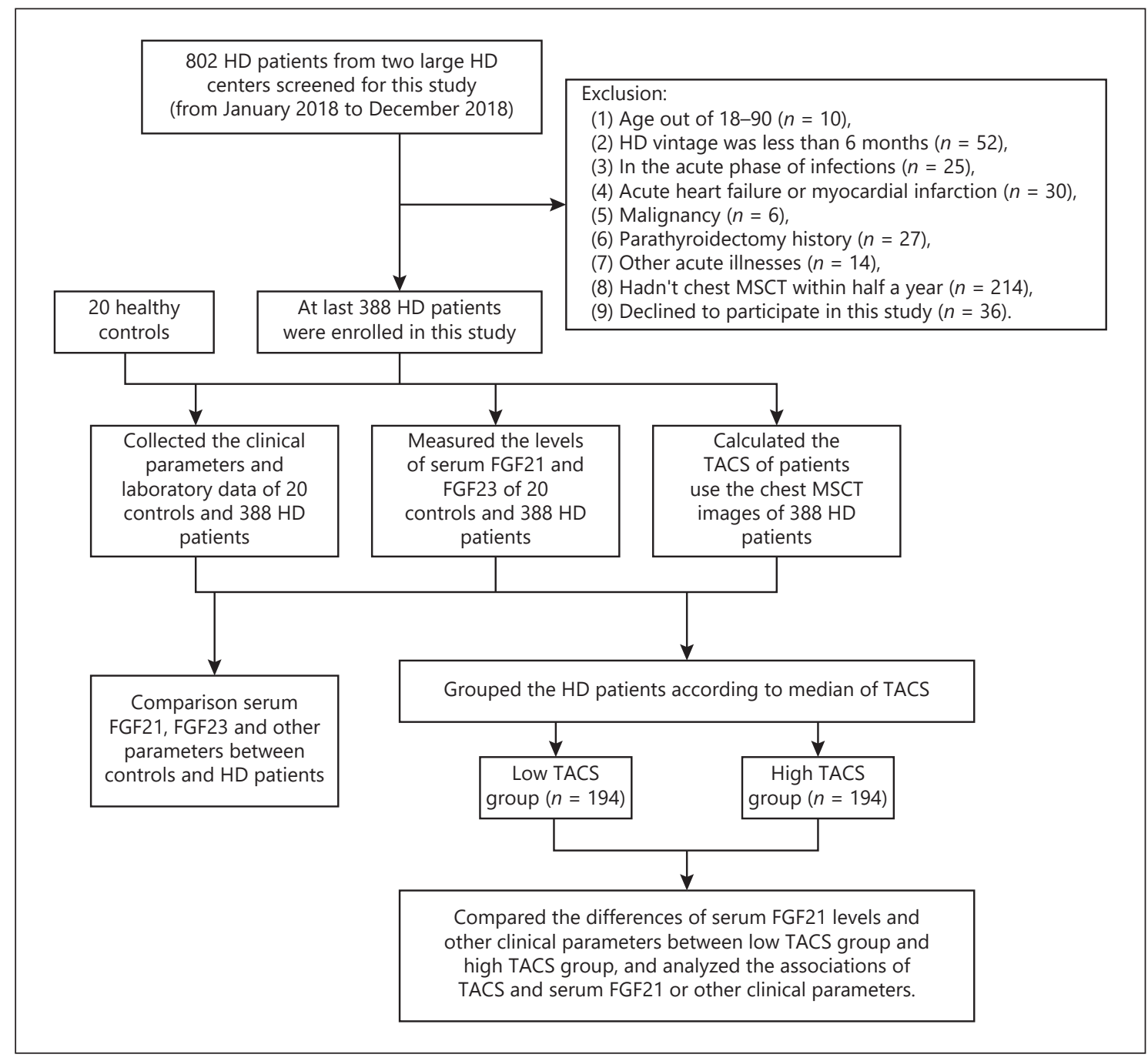

Fig. 1. Flowchart of the cross-sectional study. FGF21, fibroblast growth factor 21; FGF23, fibroblast growth factor 23; HD, haemodialysis; MSCT, multi-slice computed tomography; TACS, thoracic aorta calcification score.

different (Fig. 2). According to the median TACS $\left(0.77 \mathrm{~cm}^{3}\right)$ and the high-quartile TACS $\left(4.28 \mathrm{~cm}^{3}\right)$, the first panel was light VC $\left(<0.77 \mathrm{~cm}^{3}\right)$, the second panel was moderate VC $\left(0.77-4.28 \mathrm{~cm}^{3}\right)$, and the third panel was severe $\mathrm{VC}\left(>4.28 \mathrm{~cm}^{3}\right)$. We defined the light VC as the low TACS group and the moderate/severe VC as the high TACS group in our study (shown in Fig. 2). Second, according to the medians of AoACS $\left(0.43 \mathrm{~cm}^{3}\right)$ and DTACS $(0.25$ $\mathrm{cm}^{3}$ ), HD patients were divided into the low and high AoACS groups and the low and high DTACS groups, respectively. Since the median ATACS score was 0 , our HD patients were divided into the ATAC-positive group $(n=104)$ and ATAC-negative group $(n=284)$.

\section{Cell Culture and Intervention}

To answer the question of whether FGF21 could promote VC, we mainly focussed on the effect of FGF21 with or without para- thyroid hormone (PTH) co-incubation on calcium deposition and the EndMT process in cultured human aortic endothelial cells (HAECs). We determined the concentration of FGF21 to stimulate HAECs using a Cell Counting Kit-8 (CCK-8; Dojindo, Japan) assay. Cell culture and intervention protocols are provided in the online suppl. material.

\section{Calcium Deposition Staining for Osteoblast Differentiation}

The experiment of the calcium deposition in this study is described in the online suppl. material.

qPCR for EndMT Assays

CD31 is an endothelial cell marker, whereas RUNX2 is an osteoblast marker and FSP1 is a mesenchymal cell marker. A detailed description of the qPCR for EndMT assay in our study is provided in the online suppl. material. 

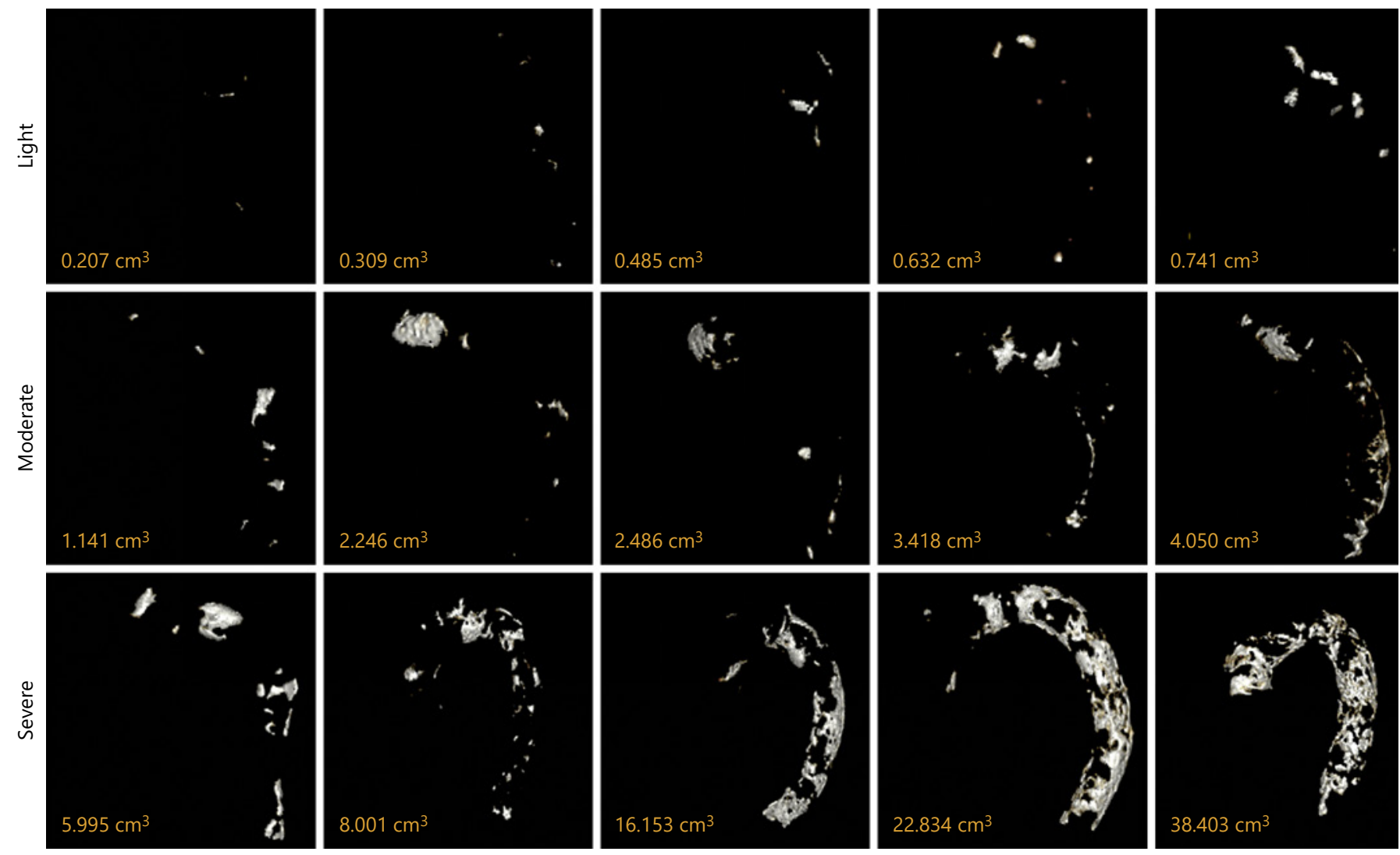

Fig. 2. Three-dimensional images of the TAC and different extents of TAC. The first panel: light VC; the second panel: moderate VC; and the third panel: severe VC. TAC, thoracic aorta calcification; VC, vascular calcification.

\section{Statistical Analyses}

Continuous variables were expressed as mean \pm SD or median with interquartile range (25-75th percentile). Categorical variables were expressed as percentages. Univariate analyses were performed to compare the differences between the 2 groups. Student's $t$ test was used to compare normally distributed data, while the Mann-Whitney U test was used for non-normally distributed data. Categorical data were compared using the $\chi^{2}$ test. Bivariate correlation analyses were performed to assess the correlation of TACS with serum FGF21 and other clinical parameters. Pearson's correlation analyses were performed for normally distributed data, and Spearman's correlation analyses were performed for non-normally distributed data. Covariance analyses were used to eliminate the influence of age on TACS. Since FGF21, FGF23, intact PTH (iPTH), HD vintage, TACS, ATACS, AoACS, and DTACS were non-normally distributed variables, these variables were taken as the logarithm for linear regression analyses. Stepwise multivariate linear regression analyses were performed to evaluate variables independently associated with TACS. Receiver operating characteristic (ROC) curves were constructed to calculate the area under the curve (AUC) and compare the prognostic value of every independently associated factor or united factor to VC. All analyses were 2 -tailed, and $p<0.05$ was considered to be statistically significant. SPSS Software, version 18.0, was used for all statistical analyses.

\section{Results}

\section{Comparison of Clinical and Laboratory}

Characteristics of HD Patients with Low or High

\section{TACS}

The age of HD patients was $57 \pm 16$ years, and 218 of the patients $(56.1 \%)$ were male. The overall prevalence of TAC in our HD patients was $70.1 \%$ (272 of 388 patients). Serum FGF21 levels in the high TACS group were significantly increased compared to those in the low TACS group, and HD patients in the high TACS group were older, had lower diastolic BP, lower uric acid, higher bicarbonate, higher $\mathrm{PPTH}$, higher FGF23, longer dialysis vintage, a higher incidence of hypertension, and a higher incidence of CVD than HD patients in the low TACS group (all $p<0.05$, Table 1). There were no additional parameters with significant differences between the 2 groups (all $p>0.05$ ). 
Table 1. Characteristics of subjects and comparison of clinical parameters and laboratory data of HD patients between the low TACS group and the high TACS group by univariate analyses

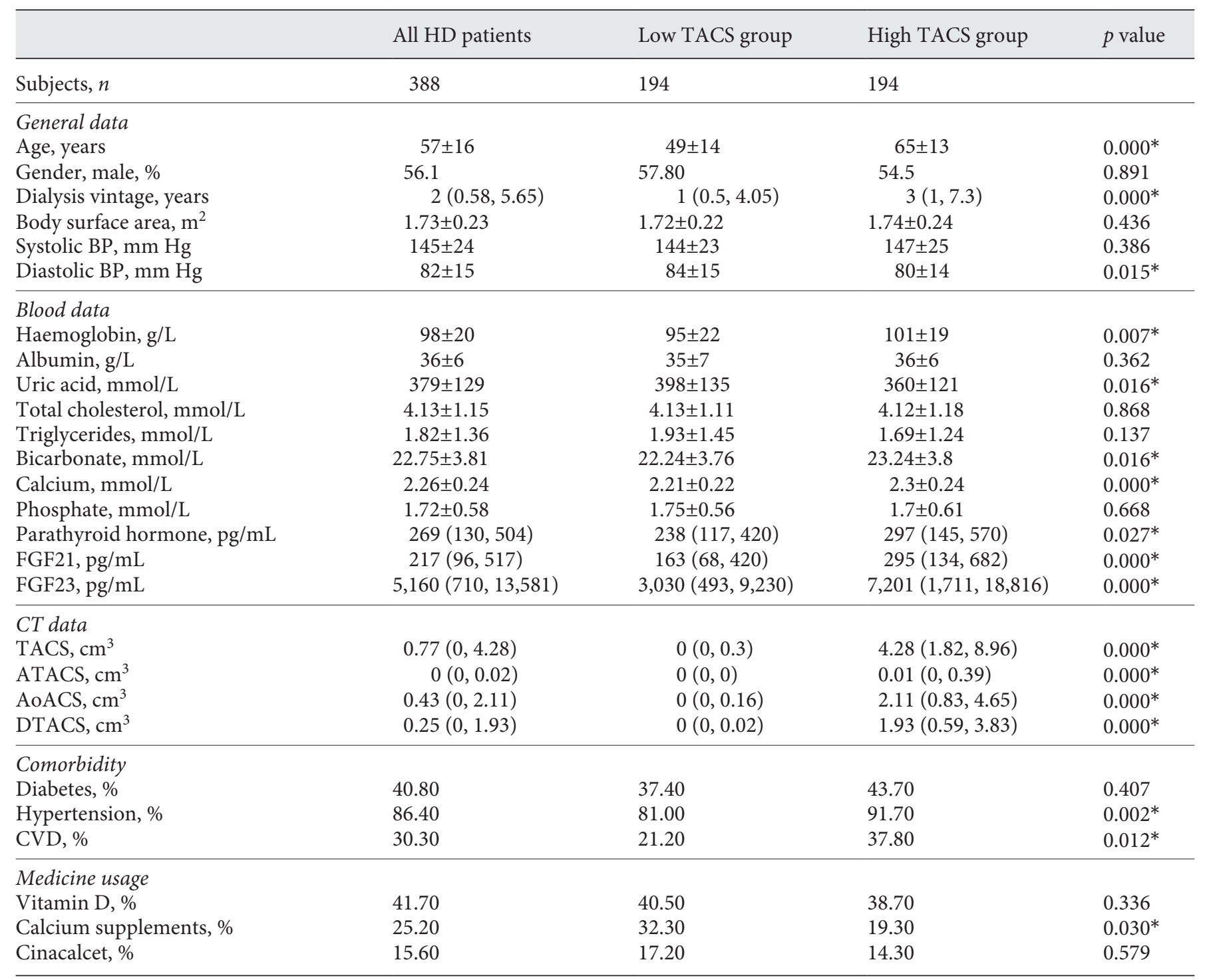

HD, haemodialysis; FGF21, fibroblast growth factor 21; FGF23, fibroblast growth factor 23; BP, blood pressure; TACS, thoracic aorta calcification score; ATACS, ascending thoracic aorta calcification score; AoACS, aortic arch calcification score; DTACS, descending thoracic aorta calcification score; CVD, cardiovascular disease. * Differences between the low TACS group and the high TACS group $(p<0.05)$.

\section{Correlation of VC with Serum FGF21 Levels and}

Other Variables in HD Patients

Serum FGF21 levels and TACS and iPTH were nonnormally distributed data and were logarithmically transformed into $\operatorname{Ln}(\mathrm{FGF} 21)$ and $\operatorname{Ln}(\mathrm{TACS}+1)$ and $\mathrm{Ln}(\mathrm{iPTH})$. Similarly, the ATACS, AoACS, and DTACS were converted into $\operatorname{Ln}(\mathrm{ATACS}+1), \quad \operatorname{Ln}(\mathrm{AoACS}+1), \quad$ and
Ln(DTACS+1), respectively. $\operatorname{Ln}(\mathrm{FGF} 21)$ was significantly positively associated with $\operatorname{Ln}(\mathrm{TACS}+1), \operatorname{Ln}(\mathrm{ATACS}+1)$, $\operatorname{Ln}($ AoACS +1$)$, and $\operatorname{Ln}(\mathrm{DTACS}+1)($ all $p<0.001$, Fig. 3 ). In addition, TACS, ATACS, AoACS, and DTACS were all correlated with age, dialysis vintage, diastolic BP, uric acid, calcium, iPTH, and FGF23 (all $p<0.05$, Table 2). 


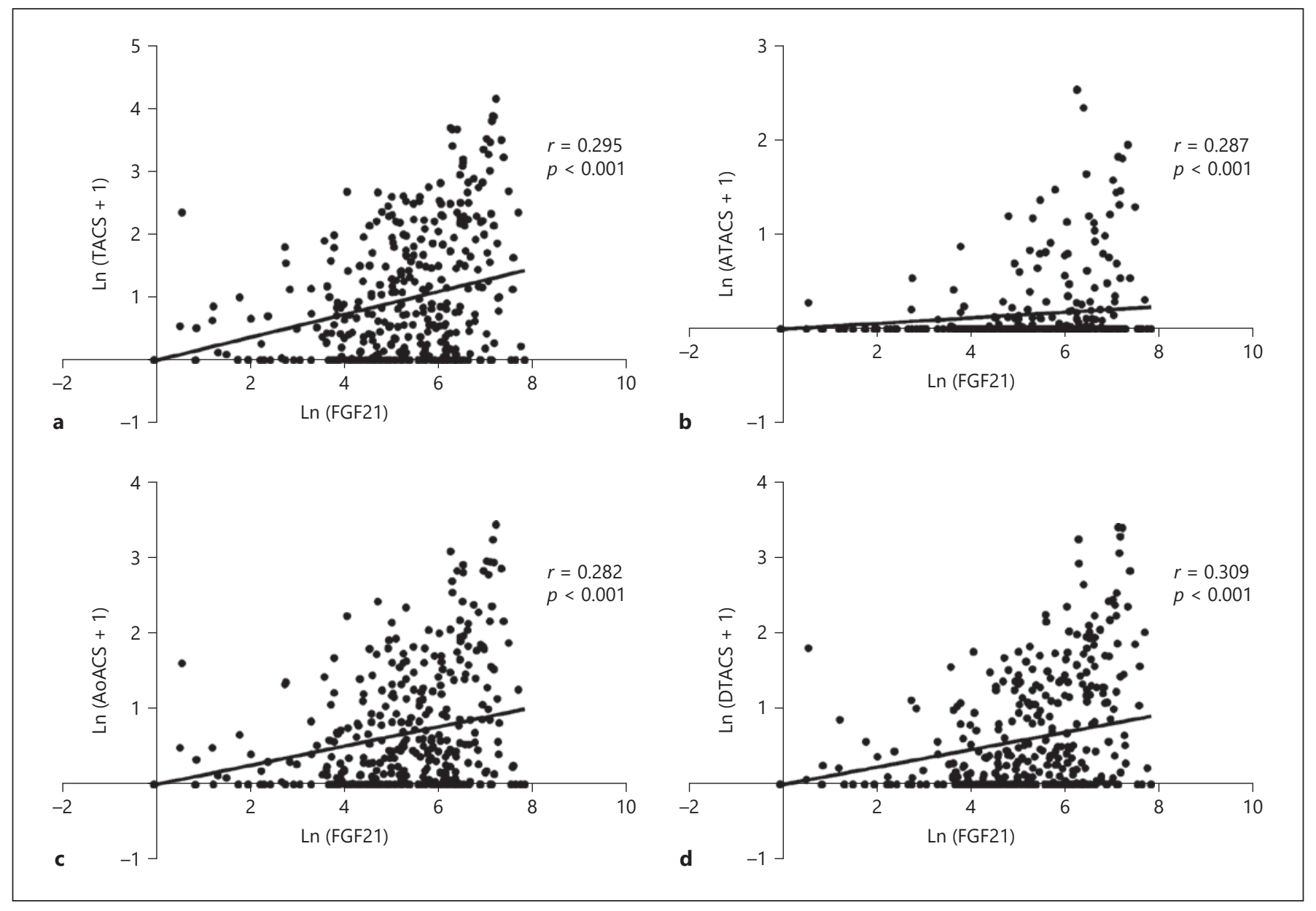

Fig. 3. Correlation of serum FGF21 with calcification scores of the whole and 3 segmented thoracic aortas in HD patients. Ln(FGF21) was significantly positively correlated with $\operatorname{Ln}(\mathrm{TACS}+1)(\mathbf{a})$, $\operatorname{Ln}(\mathrm{ATACS}+1)(\mathbf{b}), \operatorname{Ln}(\mathrm{AoACS}+1)(\mathbf{c})$, and $\operatorname{Ln}(\mathrm{DTACS}+1)(\mathbf{d})$. TACS, thoracic aorta calcification scores; ATACS, ascending tho-

Comparison of Serum FGF21 between the ATAC-

Positive Group and the ATAC-Negative Group, the High AoACS Group and the Low AoACS Group, and the High DTACS Group and the Low DTACS Group

In our HD patients, serum FGF21 levels were significantly higher in the ATAC-positive group (median 433 vs. $182 \mathrm{pg} / \mathrm{mL}$ ), the high AoACS group (median 305 vs. $159 \mathrm{pg} / \mathrm{mL}$ ), and the high DTACS group (median 298 vs. $173 \mathrm{pg} / \mathrm{mL}$ ) than in the ATAC-negative group, the low AoACS group, and the low DTACS group, respectively (all $p<0.001$, shown in Fig. 4a-c). racic aorta calcification scores; AoACS, aortic arch calcification scores; DTACS, descending thoracic aorta calcification scores; FGF21, fibroblast growth factor 21; Ln, logarithmic transformation.

Multivariate Linear Regression Analyses for the Establishment of Independent Associated Factors for VC

Variables that were different between the high and the low TACS group in the univariate analyses (age, dialysis vintage, diastolic BP, haemoglobin, uric acid, bicarbonate, calcium, iPTH, FGF21, FGF23, hypertension history, CVD history, and calcium supplement usage) and those that were well known as promoters of VC (total cholesterol, triglycerides, phosphate, and diabetes history) were entered into a multivariate linear regression analysis. The results showed that age $(\beta=0.036), \operatorname{Ln}($ FGF21) $(\beta=$ $0.192)$, calcium $(\beta=0.916)$, and $\operatorname{Ln}(\mathrm{iPTH})(\beta=0.193)$ were factors independently associated with $\operatorname{Ln}(\mathrm{TACS}+1)$ in HD patients (all $p<0.001$, Table 3 ). In addition, age, 
Table 2. Bivariate correlation analyses for the correlations of calcification scores of the whole and 3 segments of the thoracic aorta (including TACS, ATACS, AoACS, and DTACS) with other variables in HD patients

\begin{tabular}{|c|c|c|c|c|c|c|c|c|}
\hline Variable & \multicolumn{2}{|l|}{ TACS } & \multicolumn{2}{|l|}{ ATACS } & \multicolumn{2}{|l|}{ AoACS } & \multicolumn{2}{|c|}{ DTACS } \\
\hline Dialysis vintage & 0.308 & $0.000^{*}$ & 0.210 & $0.000 *$ & 0.296 & $0.000^{*}$ & 0.296 & $0.000 *$ \\
\hline BSA & 0.063 & 0.237 & 0.056 & 0.288 & 0.086 & 0.103 & 0.036 & 0.503 \\
\hline SBP & 0.082 & 0.108 & 0.032 & 0.531 & 0.092 & 0.071 & 0.079 & 0.123 \\
\hline Albumin & 0.082 & 0.114 & 0.130 & $0.012^{*}$ & 0.068 & 0.186 & 0.008 & 0.119 \\
\hline Uric acid & -0.154 & $0.003^{*}$ & -0.073 & 0.157 & -0.178 & $0.001^{*}$ & -0.117 & $0.024^{*}$ \\
\hline Total cholesterol & -0.021 & 0.681 & -0.031 & 0.552 & -0.046 & 0.371 & -0.001 & 0.998 \\
\hline Triglycerides & -0.074 & 0.154 & -0.028 & 0.628 & -0.090 & 0.080 & -0.050 & 0.337 \\
\hline Bicarbonate & 0.009 & 0.082 & 0.049 & 0.347 & 0.103 & $0.045^{*}$ & 0.195 & $0.023 *$ \\
\hline
\end{tabular}

BSA, body surface area; SBP, systolic blood pressure; DBP, diastolic blood pressure; PTH, parathyroid hormone; FGF23, fibroblast growth factor 23; TACS, thoracic aorta calcification scores; ATACS, ascending thoracic aorta calcification scores; AoACS, aortic arch calcification scores; DTACS, descending thoracic aorta calcification scores. * Correlation between the two variables $(p<0.05)$.

Table 3. Multivariate linear regression analyses for the establishment of factors independently associated with TACS

\begin{tabular}{lll}
\hline Variable & $\beta(95 \%$ CI $)$ & $p$ value \\
\hline Age & $0.036(0.030-0.042)$ & $0.000^{*}$ \\
Ln(FGF21) & $0.192(0.127-0.256)$ & $0.000^{*}$ \\
Calcium & $0.916(0.525-1.306)$ & $0.000^{*}$ \\
Ln(iPTH) & $0.193(0.106-0.281)$ & $0.000^{*}$ \\
\hline
\end{tabular}

TACS, thoracic aorta calcification score; iPTH, intact parathyroid hormone; FGF21, fibroblast growth factor $21 .{ }^{\star} p<0.05$.

calcium, $\operatorname{Ln}($ FGF21), and $\operatorname{Ln}(\mathrm{iPTH})$ were independently associated with $\operatorname{Ln}($ ATACS +1$)$; age, calcium, $\operatorname{Ln}($ FGF21), $\operatorname{Ln}(\mathrm{PPTH})$, and $\operatorname{Ln}(\mathrm{FGF} 23)$ were independently associated with $\operatorname{Ln}($ AoACS +1$)$; and age, calcium, $\operatorname{Ln}(\mathrm{FGF} 21)$, and Ln(FGF23) were independently associated with Ln(DTACS+1) (Table 4).

Prediction of VC by FGF21, Calcium, iPTH, and Age

To evaluate the discriminative performance of independently associated factors in the prediction of $\mathrm{VC}$, ROC curves were constructed (shown in Fig. 5). The
AUC of FGF21 for the prediction of VC was $0.63(p<$ 0.001 ) with high sensitivity (91\%) but poor specificity (32\%), which achieved statistical significance in HD patients (Table 5). The AUCs of age, calcium, and iPTH for the prediction of VC were $0.78(p<0.001), 0.60$ ( $p=$ $0.001)$, and $0.58(p=0.013)$, respectively. However, a combined model of FGF21 and age, calcium, and iPTH yielded a significant increment in the AUC $(0.84, p<$ 0.001 ) with optimal sensitivity (84\%) and specificity (71\%) (Table 5).

\section{FGF21 Aggravated the Effect of PTH on Calcium Deposition and EndMT in HAECs}

Compared to the control, the results revealed that the viability of HAECs showed no significant change while the FGF21 concentrations ranged from 5 to $50 \mathrm{ng} / \mathrm{mL}$, but were reduced significantly when the FGF21 concentrations were higher than $100 \mathrm{ng} / \mathrm{mL}$ (reduced to 0.86 at $100 \mathrm{ng} / \mathrm{mL}, p<0.05 ; 0.79$ at $250 \mathrm{ng} / \mathrm{mL}, p<0.01 ; 0.54$ at $500 \mathrm{ng} / \mathrm{mL}, p<0.01$; Fig. $6 \mathrm{a})$. One previous study demonstrated that PTH at a concentration of $10^{-8} \mathrm{~mol} / \mathrm{L}$ could induce HAEC EndMT [35]. In addition, the CCK-8 assay revealed that PTH $\left(10^{-8} \mathrm{~mol} / \mathrm{L}\right)$ alone and PTH $\left(10^{-8}\right.$ $\mathrm{mol} / \mathrm{L})+\mathrm{FGF} 21(50 \mathrm{ng} / \mathrm{mL})$ had no significant influence on HAEC viability (all $p>0.05$ ) (shown in Fig. 6a). There- 
Table 4. Multivariate linear regression models of factors independently associated with ATACS, AoACS, and DTACS in HD patients

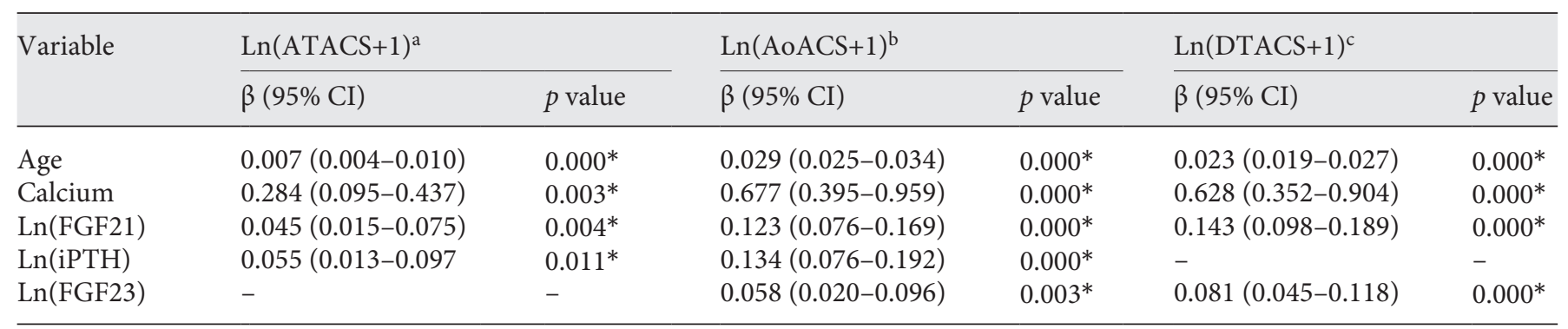

HD, haemodialysis; iPTH, intact parathyroid hormone; FGF21, fibroblast growth factor 21; FGF23, fibroblast growth factor 23; ATACS, ascending thoracic aorta calcification scores; AoACS, aortic arch calcification scores; DTACS, descending thoracic aorta calcification scores; CVD, cardiovascular disease. ${ }^{*} p<0.05$. ${ }^{\mathrm{a}-\mathrm{c}}$ All adjusted for age, dialysis vintage, diastolic BP, haemoglobin, uric acid, bicarbonate, calcium, iPTH, FGF21, FGF23, hypertension history, CVD history, calcium supplement usage, total cholesterol, triglycerides, phosphate, and diabetes history.

Fig. 4. Comparison of serum FGF21 between the ATAC-positive group and the ATAC-negative group, between the high AoACS group and the low AoACS group, and between the high DTACS group and the low DTACS group. Serum FGF21 levels were significantly higher in the ATACpositive group than in the ATAC-negative groups (a), the high AoACS group than in the low AoACS group (b), and the high DTACS group than in the low DTACS group (c). ATAC, ascending thoracic aorta calcification; AoACS, aortic arch calcification scores; DTACS, descending thoracic aorta calcification scores; FGF21, fibroblast growth factor 21 .

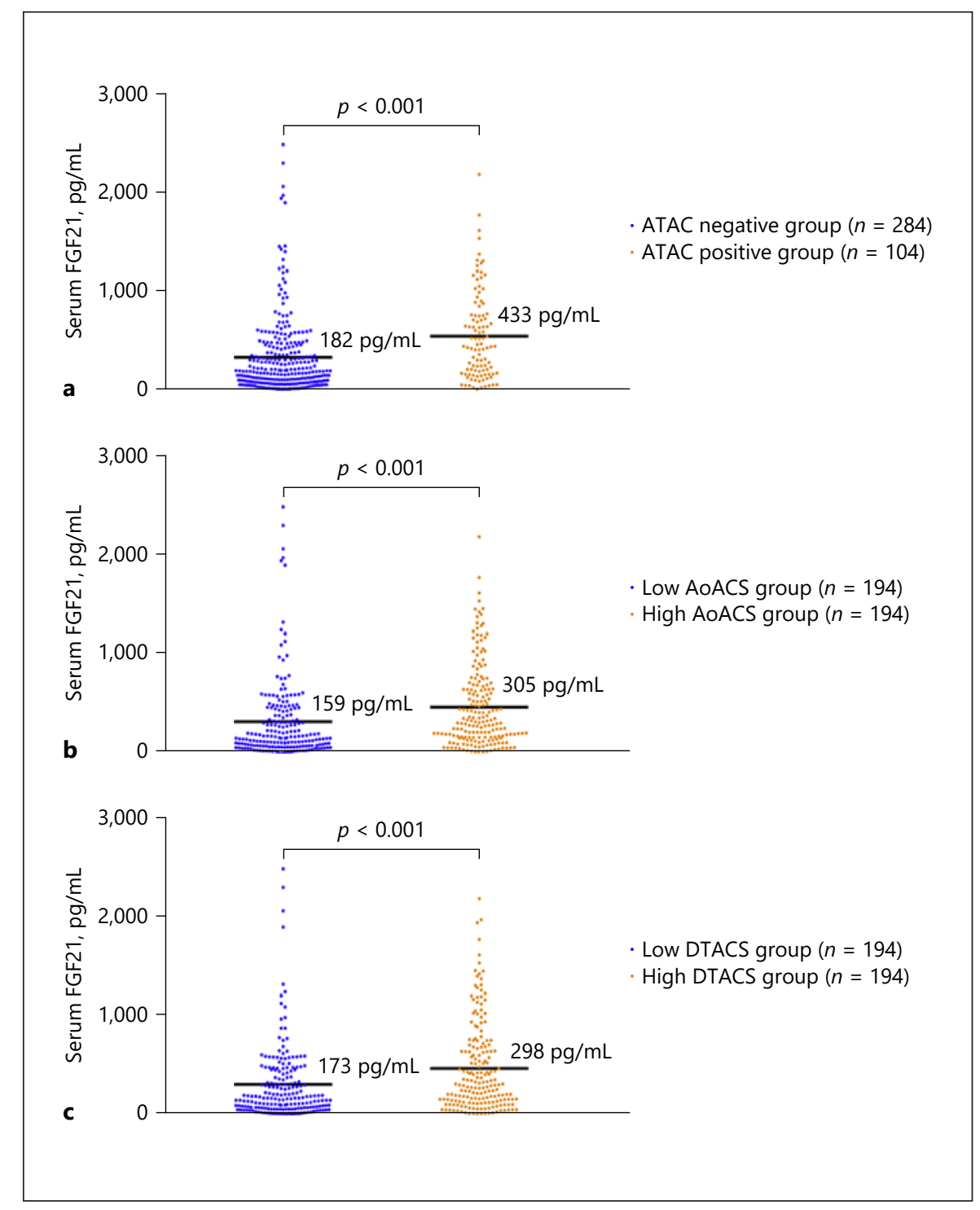


Table 5. The AUC of separated and united independently associated factors of TACS in ROC curve analyses

\begin{tabular}{lllll}
\hline Variable & AUC (95\% CI) & $p$ value & $\begin{array}{l}\text { Sensitivity, } \\
\%\end{array}$ & $\begin{array}{l}\text { Specificity, } \\
\%\end{array}$ \\
\hline Age & $0.78(0.74-0.83)$ & $0.000^{*}$ & 59 & 87 \\
FGF21 & $0.63(0.57-0.69)$ & $0.000^{*}$ & 91 & 32 \\
Calcium & $0.60(0.54-0.66)$ & $0.001^{*}$ & 92 & 22 \\
iPTH & $0.58(0.51-0.63)$ & $0.012^{*}$ & 60 & 54 \\
FGF21 + iPTH & $0.64(0.58-0.69)$ & $0.000^{*}$ & 60 & 60 \\
Age + calcium + iPTH + FGF21 & $0.84(0.79-0.87)$ & $0.000^{*}$ & 84 & 71 \\
\hline
\end{tabular}

AUC, area under the curve; ROC, receiver operating characteristic; TACS, thoracic aorta calcification score; iPTH, intact parathyroid hormone; FGF21, fibroblast growth factor $21 .^{*} p<0.05$.

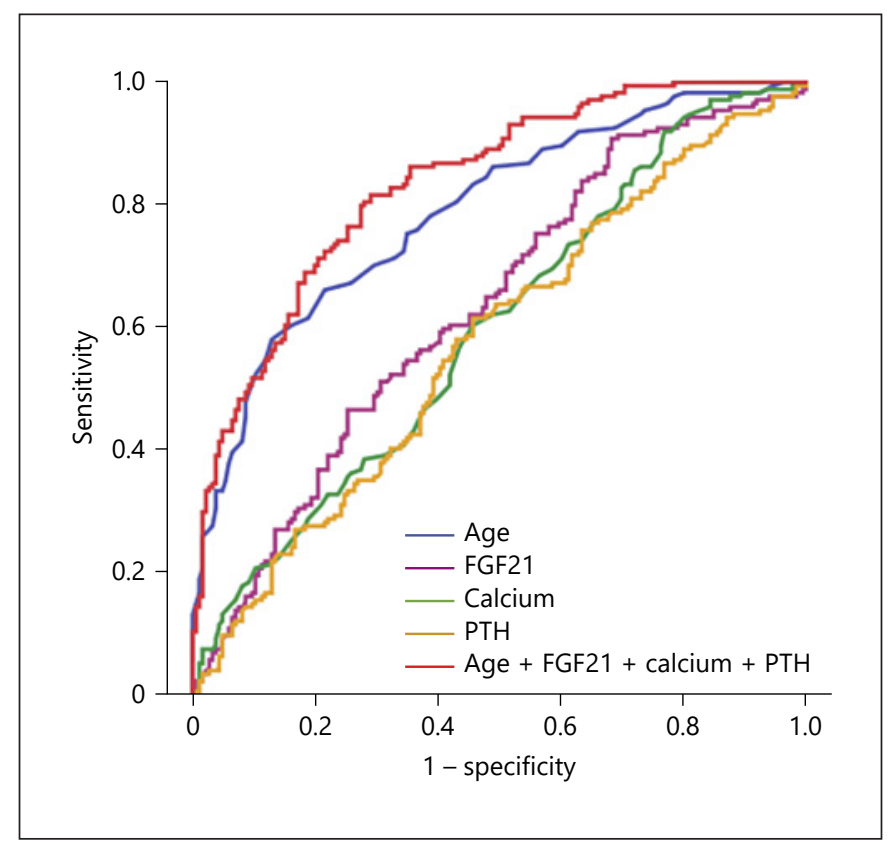

Fig. 5. Receiver operating characteristic curve analyses of age, iPTH, FGF21, calcium, and the combined model of age, FGF21, calcium, and $\mathrm{iPTH}$ for prediction of $\mathrm{VC}$ in $\mathrm{HD}$ patients. Blue line, age $(\mathrm{AUC}=0.78, p<0.001)$; purple line, FGF21 (AUC $=0.63, p<$ 0.001 ); green line, calcium (AUC $=0.60, p=0.001)$; orange line, iPTH $(\mathrm{AUC}=0.58, p=0.013)$; red line, age + FGF21 $+\mathrm{iPTH}+$ FGF23 (AUC $=0.83, p<0.001)$. FGF21, fibroblast growth factor 21; FGF23, fibroblast growth factor 23; iPTH, intact parathyroid hormone; VC, vascular calcification; HD, haemodialysis.

fore, FGF2 1 concentrations of $50 \mathrm{ng} / \mathrm{mL}, \mathrm{PTH}$ concentrations of $10^{-8} \mathrm{~mol} / \mathrm{L}$, and PTH $\left(10^{-8} \mathrm{~mol} / \mathrm{L}\right)+$ FGF21 (50 $\mathrm{ng} / \mathrm{mL}$ ) were applied in our subsequent experiments.

Alizarin Red staining (ARS) showed that calcium deposition was observed in cultured HAECs stimulated by

FGF21 and Vascular Calcification in HD Patients
PTH alone and was even aggravated by PTH + FGF21; however, no calcium deposition was observed in HAECs stimulated by FGF21 alone (shown in Fig. 6b). This result suggested that FGF21 aggravated the calcification effect of PTH on HAECs.

Next, we evaluated the effect of FGF21 on the EndMT process of HAECs. Compared with the control, reduced expression of CD31 mRNA (shown in Fig. 6c) in parallel with increased expression of RUNX2 mRNA (shown in Fig. 6d) and FSP1 mRNA (shown in Fig. 6e) was observed in HAECs stimulated by PTH. Our results indicated that PTH alone induced EndMT. However, FGF21 alone had no significant effect on the EndMT process. The stimulation of PTH + FGF21 in HAECs aggravated the effect of PTH alone on EndMT. More interestingly, these results were consistent with the ARS results mentioned above.

\section{Discussion}

To the best of our knowledge, this study is the first one which showed that serum FGF21 levels were significantly and independently correlated with VC in HD patients. Furthermore, FGF21 combined with age, calcium, and iPTH showed optimal sensitivity (84\%) and specificity (71\%) for the prediction of VC in HD patients. In addition, we first demonstrated that FGF21 aggravated the effect of PTH on calcium deposition and EndMT in HAECs. Taken together, our vivo and vitro results indicated that FGF21 was a novel predictor and potential promoter of $\mathrm{VC}$ in HD patients.

$\mathrm{CKD}$ is an international public health epidemic and increases annually [36]. The presence and extent of $\mathrm{VC}$ are notably higher in patients with CKD than in the general population, even compared with patients at high risk of 

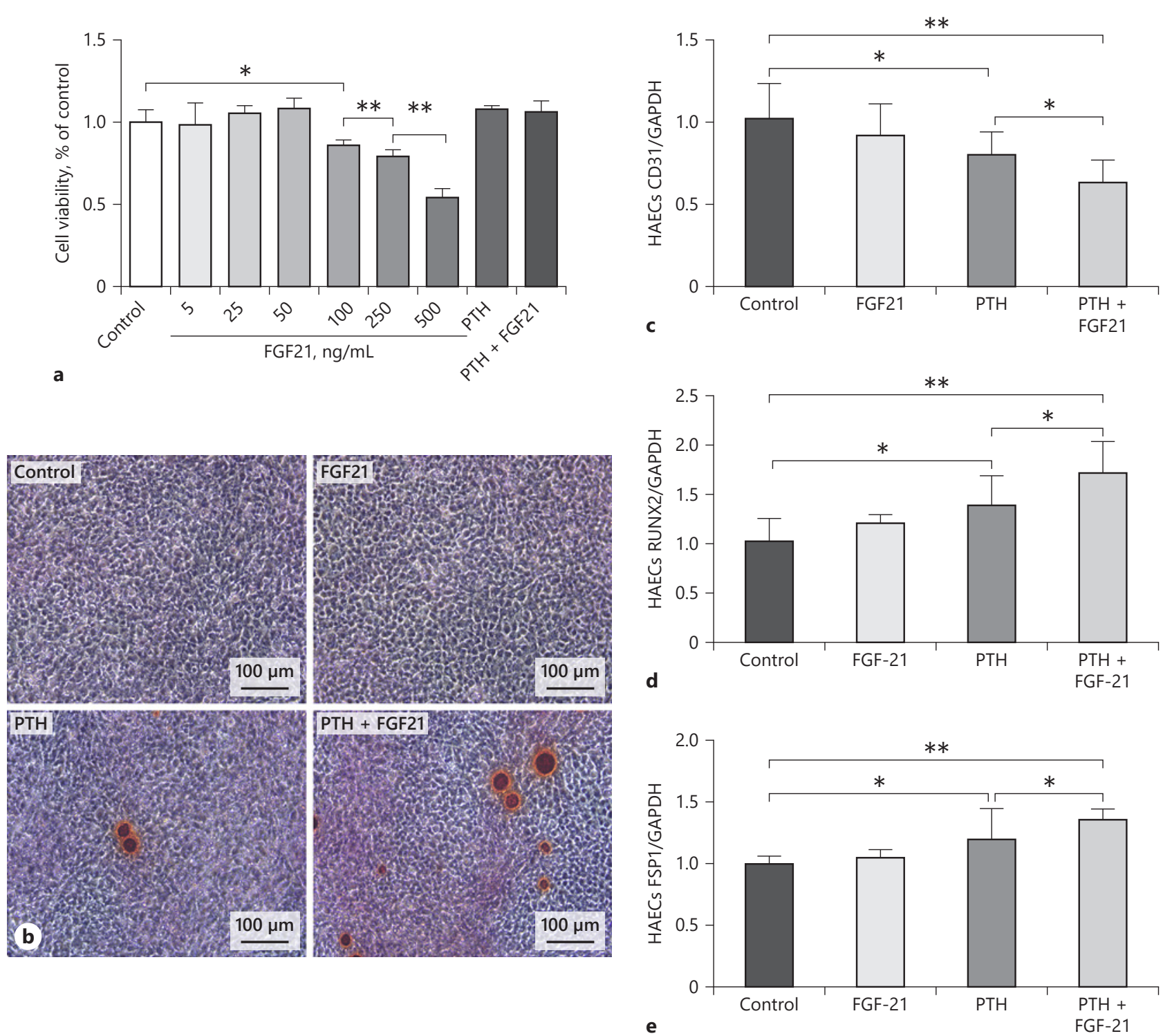

Fig. 6. FGF21 enhanced the calcification effect of PTH on HAECs via calcium deposition and EndMT. a CCK-8 assay for HAEC viability in different conditions. b Alizarin Red staining on HAECs stimulated by FGF21 with or without PTH co-incubation. Bright red or orange staining indicates a positive result for calcium deposition. c Expression of CD31 mRNA in HAECs stimulated by FGF21 with or without PTH co-incubation. d Expression of

CVD but with normal renal function [37]. TAC has been considered an independent predictor of CVD and mortality [38]. The KDIGO CKD-MBD guidelines have indicated that electron beam computed tomography and MSCT are the gold standards and the most sensitive methods for
RUNX2 mRNA in HAECs stimulated by FGF21 with or without PTH co-incubation. e Expression of FSP1 mRNA in HAECs stimulated by FGF21 with or without PTH co-incubation. ${ }^{*} p<0.05$ and ${ }^{* *} p<0.01$. EndMT, endothelial-to-mesenchymal transition; FGF21, fibroblast growth factor 21; PTH, parathyroid hormone; HAECs, human aortic endothelial cells; CCK-8, Cell Counting Kit-8.

the detection and quantification of VC [39]. In this study, we used chest MSCT for TAC evaluation and found that the prevalence of TAC in HD patients was $70.1 \%$, which was similar to other epidemiological findings [40, 41]. 
Table 6. Comparison of clinical parameters and laboratory data between HD patients and healthy controls

\begin{tabular}{lccc}
\hline & HD patients & Healthy controls & $p$ value \\
\hline Subjects, $n$ & 388 & 20 & - \\
Age, years & $57 \pm 16$ & $53 \pm 17$ & 0.136 \\
Gender, male & $56.10 \%$ & $60.00 \%$ & 0.248 \\
Dialysis vintage, years & $2(0.58,5.65)$ & - & - \\
Body mass index, kg/cm ${ }^{2}$ & $1.73 \pm 0.23$ & $1.72 \pm 0.61$ & 0.873 \\
Systolic BP, mm Hg & $145 \pm 24$ & $134 \pm 16$ & $0.023^{*}$ \\
Diastolic BP, mm Hg & $82 \pm 15$ & $76 \pm 9$ & $0.016^{*}$ \\
Haemoglobin, g/L & $98 \pm 20$ & $145.55 \pm 16.06$ & $0.000^{*}$ \\
Albumin, g/L & $36 \pm 6$ & $45.93 \pm 4.56$ & $0.000^{*}$ \\
Uric acid, mmol/L & $379 \pm 129$ & $302.45 \pm 79.40$ & $0.000^{*}$ \\
Cholesterol, mmol/L & $4.13 \pm 1.15$ & $4.69 \pm 0.85$ & $0.008^{*}$ \\
Triglycerides, mmol/L & $1.82 \pm 1.36$ & $1.25 \pm 0.72$ & $0.026^{*}$ \\
Bicarbonate, mmol/L & $22.75 \pm 3.81$ & $24.90 \pm 1.54$ & $0.000^{*}$ \\
Calcium, mmol/L & $2.26 \pm 0.24$ & $2.34 \pm 0.15$ & $0.048^{*}$ \\
Phosphate, mmol/L & $1.72 \pm 0.58$ & $1.14 \pm 0.25$ & $0.000^{*}$ \\
Intact parathyroid hormone, pg/mL & $269(130,504)$ & - & - \\
FGF21, pg/mL & $217(96,517)$ & $20(2,287)$ & $0.000^{*}$ \\
FGF23, pg/mL & $5,160(710,13,581)$ & $293(93,529)$ & $0.000^{*}$ \\
\hline
\end{tabular}

HD, haemodialysis; FGF21, fibroblast growth factor 21; FGF23, fibroblast growth factor 23. * Differences between the HD patients and the healthy controls $(p<0.05)$.

FGF21 is mainly secreted by the liver and adipose tissue and released into the circulation, functioning as a hormone capable of modulating systemic glucose and lipid metabolism [42]. Many studies have indicated that serum FGF21 levels were negatively correlated with renal function and were elevated $8-15$-folds in patients receiving dialysis in comparison to controls [21,22]. Furthermore, high serum FGF21 levels in ESRD patients predict high all-cause mortality [43]. In our study, the median serum FGF21 level in HD patients was elevated 11-fold compared with healthy controls (Table 6). Serum FGF21 levels were positively correlated with TACS in our HD patients, which suggested serum FGF21 increased with the extent of TAC in HD patients. Multivariate regression analyses showed that only age, FGF21, calcium, and iPTH were independent factors associated with TACS. Even after adjusting for the covariate of age, the independent association between serum FGF21 and TACS remained. All the aforementioned results suggested a key role of FGF21 in VC in HD patients.

Because computed tomography examination is radiologically hazardous to humans and calcification assessment software is not used universally, we recommend serum biomarkers for the prediction of $\mathrm{VC}$ in $\mathrm{HD}$ patients. Our clinical study revealed that serum FGF21 was obvi- ously increased in HD patients and was positively related to the extent of TAC. Moreover, FGF21 combined with age, calcium, and iPTH formed a combined predictor whose AUC for the prediction of $\mathrm{VC}$ in $\mathrm{HD}$ patients was $0.84(p<0.001)$ with high sensitivity and specificity. Taken together, these results indicate that the detection of serum FGF21 with age, calcium, and iPTH could be used as a preliminary screening method for the prediction of $\mathrm{VC}$ in HD patients. This discovery can provide an easier way to identify $\mathrm{HD}$ patients with moderate/severe VC and can avoid extra radiation and cost. It may also provide a new therapeutic target for the treatment of VC in HD patients.

A study compared HD patients and sex-age-matched subjects without CKD revealed that HD patients were more likely to exhibit a greater arc of calcification at the culprit of VC - a higher frequency of intimal thin calcium and calcified nodules [44]. Secondary hyperparathyroidism (SHPT) is a common complication of CKD patients [45]. In a study of nearly 1300 US dialysis centres and $39,000 \mathrm{HD}$ patients, $>11 \%$ of patients had an $\mathrm{PTTH}$ of $>600 \mathrm{pg} / \mathrm{mL}$ [46]. PTH could induce the transition of ECs to chondrogenic cells via EndMT [47]. In our vitro study, FGF21 alone could not induce EndMT and calcium deposition in HAECs, but when FGF21 co-incubated with 
$\mathrm{PTH}$, these 2 processes were aggravated compared with PTH incubated alone, suggesting that FGF21 was a potential promoter of intimal calcification in an environment where SHPT coexisted. Cao Fang reported that FGF21 attenuates the calcification of vascular smooth muscle cells (VSMCs) in vitro [48], but our study revealed that FGF21 can aggravate the calcification capacity of PTH on HAECs, which suggested that the pro-calcification effects of FGF21 on ECs could overcome its anticalcification effects on VSMCs. This is further confirmed by our clinical data that FGF21 combined with iPTH is better for the prediction of $\mathrm{VC}$ than iPTH alone (online suppl. Table 2). Hence, this study provided both in vivo and in vitro evidence that FGF21 was one of the key factors that predicted $\mathrm{VC}$ and promoted intimal calcification in HD patients.

The most important significance and contributions of this study are mainly in 3 aspects. First, we screened 388 of $802 \mathrm{HD}$ patients in 2 large HD centres in China, comprehensively evaluated the calcification levels of the whole thoracic aorta and different segments of the thoracic aorta, and, for the first time, found an independent correlation of FGF21 with VC. Second, FGF21 combined with age, calcium, and $\mathrm{PTH}$ exerted a high AUC with high sensitivity and specificity for the prediction of $\mathrm{VC}$ in $\mathrm{HD}$ patients. Third, our results indicated for the first time that FGF21 can amplify the role of PTH in promoting calcium deposition and EndMT in vitro, further supporting the results of our clinical data.

Despite the added precautions, there are limitations to the current study. First, the relatively small sample size and the cross-sectional study design preclude the determination of cause and effect. This limitation highlights the need for adequately powered RCTs and observational studies to further confirm the findings presented here. Second, because the clinical results may differ owing to the ethnicity of the participants, our results do not extend to other ethnic groups. Third, our study is a partly retrospective study and all the HD patients in our study had MSCT scans before the study, which may lead to a high risk of selection bias. The signalling pathway and detailed mechanism of FGF21 combined with PTH to promote calcium deposition and EndMT merit further study.

\section{Conclusions}

We reported for the first time that serum FGF21 contributes to $\mathrm{VC}$ and may serve as a novel predictor for the presence and extent of TAC in HD patients. Further-

more, FGF21 enhanced the calcification effect of PTH on HAECs. These findings provide novel insight into FGF21 on VC in HD patients and may indicate a new therapeutic target for this life-threatening disorder.

\section{Acknowledgements}

The authors are grateful to all participants for their time and effort.

\section{Statement of Ethics}

This study was performed in accordance with the Declaration of Helsinki and was approved by the Ethics Committee of Zhongda Hospital, Affiliated to Southeast University (Approval No. 2019ZDKYSB191, August 5, 2019). All patients have given their written informed consent.

\section{Conflict of Interest Statement}

None of the authors has conflicts of interest to declare.

\section{Funding Sources}

This work was supported by grants from the National Natural Science Foundation of China (81700618), the Natural Science Foundation of Jiangsu Province (BK20181487), a Southeast University High-Level Thesis Project, the Clinical Medical Research Fund of Chinese Medical Association, and the CKD-MBD Youth Research Fund to Bin Wang; the Medical Research Project of Jiangsu Commission of Health (H2019061); the National Key Research Programme (2018YFC130046 and 2018YFC1314000) and the Clinic Research Centre of Jiangsu Province (BL2014080); the Key Subject Construction Programme for Nephrology of Suzhou (Szxk201807); and Jiangsu Provincial Health and Family Planning Commission (KY2018105).

\section{Availability of Data and Material}

The datasets used and/or analyzed during the current study are available from the corresponding author on reasonable request and approval by the principal investigator.

References

Kidney Dis 2021;7:227-240

DOI: $10.1159 / 000512750$
1 Polkinghorne KR. ESKD or cancer: given the choice, which would you rather have? Am J Kidney Dis. 2019 Jun;73(6):753-5.

2 Hundemer GL, Sood MM. Predialysis care and cardiovascular outcomes: why the lead up to dialysis matters. Kidney Int Rep. 2019 May; $4(5): 635-7$. 
3 Moe S, Drüeke T, Cunningham J, Goodman W, Martin K, Olgaard K, et al. Definition, evaluation, and classification of renal osteodystrophy: a position statement from Kidney Disease: Improving Global Outcomes (KDIGO). Kidney Int. 2006 Jun;69(11):1945-53.

4 Raggi P, Kleerekoper M. Contribution of bone and mineral abnormalities to cardiovascular disease in patients with chronic kidney disease. Clin J Am Soc Nephrol. 2008 May; 3(3):836-43.

5 Foley RN, Murray AM, Li S, Herzog CA, McBean AM, Eggers PW, et al. Chronic kidney disease and the risk for cardiovascular disease, renal replacement, and death in the United States Medicare population, 1998 to 1999. J Am Soc Nephrol. 2005 Feb;16(2):489-95.

6 Qunibi WY, Abouzahr F, Mizani MR, Nolan CR, Arya R, Hunt KJ. Cardiovascular calcification in Hispanic Americans (HA) with chronic kidney disease (CKD) due to type 2 diabetes. Kidney Int. 2005 Jul;68(1):271-7.

7 Kraus MA, Kalra PA, Hunter J, Menoyo J, Stankus N. The prevalence of vascular calcification in patients with end-stage renal disease on hemodialysis: a cross-sectional observational study. Ther Adv Chronic Dis. 2015 May;6(3):84-96.

8 Coen G, Pierantozzi A, Spizzichino D, Sardella D, Mantella D, Manni M, et al. Risk factors of one year increment of coronary calcifications and survival in hemodialysis patients. BMC Nephrol. 2010 Jun 21;11:10.

9 Shroff R, Long DA, Shanahan C. Mechanistic insights into vascular calcification in CKD. J Am Soc Nephrol. 2013 Feb;24(2):179-89.

10 Itoh N. Hormone-like (endocrine) Fgfs: their evolutionary history and roles in development, metabolism, and disease. Cell Tissue Res. 2010 Oct;342(1):1-11.

11 Goetz R, Beenken A, Ibrahimi OA, Kalinina J, Olsen SK, Eliseenkova AV, et al. Molecular insights into the klotho-dependent, endocrine mode of action of fibroblast growth factor 19 subfamily members. Mol Cell Biol. 2007 May;27(9):3417-28.

12 Gutierrez OM, Mannstadt M, Isakova T, Rauh-Hain JA, Tamez H, Shah A, et al. Fibroblast growth factor 23 and mortality among patients undergoing hemodialysis. $\mathrm{N}$ Engl $\mathrm{J}$ Med. 2008 Aug 7;359(6):584-92.

13 Jean G, Terrat JC, Vanel T, Hurot JM, Lorriaux C, Mayor B, et al. High levels of serum fibroblast growth factor (FGF)-23 are associated with increased mortality in long haemodialysis patients. Nephrol Dial Transplant. 2009 Sep;24(9):2792-6.

14 Isakova $\mathrm{T}, \mathrm{Xie} \mathrm{H}$, Yang W, Xie D, Anderson $\mathrm{AH}$, Scialla J, et al. Fibroblast growth factor 23 and risks of mortality and end-stage renal disease in patients with chronic kidney disease. JAMA. 2011 Jun 15;305(23):2432-9.
15 Nasrallah MM, El-Shehaby AR, Salem MM, Osman NA, El Sheikh E, Sharaf El Din UA. Fibroblast growth factor-23 (FGF-23) is independently correlated to aortic calcification in haemodialysis patients. Nephrol Dial Transplant. 2010 Aug;25(8):2679-85.

16 Desjardins L, Liabeuf S, Renard C, Lenglet A, Lemke HD, Choukroun G, et al. FGF23 is independently associated with vascular calcification but not bone mineral density in patients at various CKD stages. Osteoporos Int. 2012 Jul;23(7):2017-25.

17 Lindberg K, Olauson H, Amin R, Ponnusamy A, Goetz R, Taylor RF, et al. Arterial klotho expression and FGF23 effects on vascular calcification and function. PLoS One. 2013;8(4): e60658.

18 Scialla JJ, Lau WL, Reilly MP, Isakova T, Yang $\mathrm{HY}$, Crouthamel MH, et al. Fibroblast growth factor 23 is not associated with and does not induce arterial calcification. Kidney Int. 2013 Jun;83(6):1159-68.

19 Zhu D, Mackenzie NC, Millan JL, Farquharson C, MacRae VE. A protective role for FGF23 in local defence against disrupted arterial wall integrity? Mol Cell Endocrinol. 2013 Jun 15;372(1-2):1-11.

20 Chen YX, Huang C, Duan ZB, Xu CY, Chen Y. Klotho/FGF23 axis mediates high phosphate-induced vascular calcification in vascular smooth muscle cells via $\mathrm{Wnt} 7 \mathrm{~b} / \beta$-catenin pathway. Kaohsiung J Med Sci. 2019 Jul; 35(7):393-400

21 Stein S, Bachmann A, Lössner U, Kratzsch J, Blüher M, Stumvoll M, et al. Serum levels of the adipokine FGF21 depend on renal function. Diabetes Care. 2009 Jan;32(1):126-8.

22 Anuwatmatee S, Tang S, Wu BJ, Rye KA, Ong KL. Fibroblast growth factor 21 in chronic kidney disease. Clin Chim Acta. 2019 Feb; 489:196-202.

23 Staiger H, Keuper M, Berti L, Hrabe de Angelis M, Häring HU. Fibroblast growth factor 21-metabolic role in mice and men. Endocr Rev. 2017 Oct 1;38(5):468-88.

24 Coskun T, Bina HA, Schneider MA, Dunbar JD, Hu CC, Chen Y, et al. Fibroblast growth factor 21 corrects obesity in mice. Endocrinology. 2008 Dec;149(12):6018-27.

25 Kharitonenkov A, Shiyanova TL, Koester A, Ford AM, Micanovic R, Galbreath EJ, et al. FGF-21 as a novel metabolic regulator. J Clin Invest. 2005 Jun;115(6):1627-35.

26 Xu J, Lloyd DJ, Hale C, Stanislaus S, Chen M, Sivits G, et al. Fibroblast growth factor 21 reverses hepatic steatosis, increases energy expenditure, and improves insulin sensitivity in diet-induced obese mice. Diabetes. 2009 Jan; 58(1):250-9.

27 Fazeli PK, Faje AT, Cross EJ, Lee H, Rosen CJ, Bouxsein ML, et al. Serum FGF-21 levels are associated with worsened radial trabecular bone microarchitecture and decreased radial bone strength in women with anorexia nervosa. Bone. 2015 Aug;77:6-11.
28 Li ZC, Xiao J, Wang G, Li MQ, Hu KZ, Ma T, et al. Fibroblast growth factor-21 concentration in serum and synovial fluid is associated with radiographic bone loss of knee osteoarthritis. Scand J Clin Lab Invest. 2015 Apr; 75(2):121-5.

29 Talukdar S, Zhou Y, Li D, Rossulek M, Dong $\mathrm{J}$, Somayaji V, et al. A long-acting FGF21 molecule, PF-05231023, decreases body weight and improves lipid profile in non-human primates and type 2 diabetic subjects. Cell Metab. 2016 Mar 8;23(3):427-40.

30 Kim AM, Somayaji VR, Dong JQ, Rolph TP, Weng Y, Chabot JR, et al. Once-weekly administration of a long-acting fibroblast growth factor 21 analogue modulates lipids, bone turnover markers, blood pressure and body weight differently in obese people with hypertriglyceridaemia and in non-human primates. Diabetes Obes Metab. 2017 Dec; 19(12):1762-72.

31 Farhat GN, Newman AB, Sutton-Tyrrell K, Matthews KA, Boudreau R, Schwartz AV, et al. The association of bone mineral density measures with incident cardiovascular disease in older adults. Osteoporos Int. $2007 \mathrm{Jul}$; 18(7):999-1008.

32 Cai G, Keen HI, Host LV, Aitken D, Laslett LL, Winzenberg T, et al. Once-yearly zoledronic acid and change in abdominal aortic calcification over 3 years in postmenopausal women with osteoporosis: results from the HORIZON Pivotal Fracture Trial. Osteoporosis Int. $2020 \mathrm{Sep} ; 31(9)$ :1714-47.

33 Chen PY, Qin L, Baeyens N, Li G, Afolabi T, Budatha M, et al. Endothelial-to-mesenchymal transition drives atherosclerosis progression. J Clin Invest. 2015 Oct 26;125(12):451428.

34 Hong L, Du X, Li W, Mao Y, Sun L, Li X. EndMT: a promising and controversial field. Eur J Cell Biol. 2018 Sep;97(7):493-500.

35 Wu M, Zhang JD, Tang RN, Crowley SD, Liu $\mathrm{H}, \mathrm{Lv} \mathrm{LL}$, et al. Elevated PTH induces endothelial-to-chondrogenic transition in aortic endothelial cells. Am J Physiol Renal Physiol. 2017 Mar 1;312(3):F436-44

36 Coresh J, Selvin E, Stevens LA, Manzi J, Kusek JW, Eggers P, et al. Prevalence of chronic kidney disease in the United States. JAMA. 2007 Nov 7;298(17):2038-47.

37 Braun J, Oldendorf M, Moshage W, Heidler R, Zeitler E, Luft FC. Electron beam computed tomography in the evaluation of cardiac calcification in chronic dialysis patients. Am J Kidney Dis. 1996 Mar;27(3):394-401.

38 Eisen A, Tenenbaum A, Koren-Morag N, Tanne D, Shemesh J, Imazio M, et al. Calcification of the thoracic aorta as detected by spiral computed tomography among stable angina pectoris patients: association with cardiovascular events and death. Circulation. 2008 Sep 23;118(13):1328-34. 
39 Kidney Disease: Improving Global Outcomes CKDMBDWG. KDIGO clinical practice guideline for the diagnosis, evaluation, prevention, and treatment of Chronic Kidney Disease-Mineral and Bone Disorder (CKDMBD). Kidney Int Suppl. 2009 Aug;(113):S1130.

40 Adeney KL, Siscovick DS, Ix JH, Seliger SL, Shlipak MG, Jenny NS, et al. Association of serum phosphate with vascular and valvular calcification in moderate CKD. J Am Soc Nephrol. 2009 Feb;20(2):381-7.

41 Liu ZH, Yu XQ, Yang JW, Jiang AL, Liu BC, Xing CY, et al. Prevalence and risk factors for vascular calcification in Chinese patients receiving dialysis: baseline results from a prospective cohort study. Curr Med Res Opin. 2018 Aug;34(8):1491-500.
42 Adams AC, Kharitonenkov A. FGF21: the center of a transcriptional nexus in metabolic regulation. Curr Diabetes Rev. 2012 Jul 1;8(4): 285-93.

43 Kohara M, Masuda T, Shiizaki K, Akimoto T, Watanabe Y, Honma S, et al. Association between circulating fibroblast growth factor 21 and mortality in end-stage renal disease. PLoS One. 2017;12(6):e0178971.

44 Chin CY, Matsumura M, Maehara A, Zhang W, Lee CT, Yamamoto MH, et al. Coronary plaque characteristics in hemodialysis-dependent patients as assessed by optical coherence tomography. Am J Cardiol. 2017 May 1; 119(9):1313-9.
45 Komaba H, Kakuta T, Fukagawa M. Management of secondary hyperparathyroidism: how and why? Clin Exp Nephrol. 2017 Mar; 21(Suppl 1):37-45.

46 Block GA, Yusuf AA, Danese MD, Wirtz HS, $\mathrm{Hu}$ Y, Do TP, et al. Facility-level CKD-MBD composite score and risk of adverse clinical outcomes among patients on hemodialysis. BMC Nephrol. 2016 Nov 4;17(1):166.

$47 \mathrm{Wu}$ M, Tang RN, Liu H, Pan MM, Liu BC. Cinacalcet ameliorates aortic calcification in uremic rats via suppression of endothelial-tomesenchymal transition. Acta Pharmacol Sin. 2016 Nov;37(11):1423-31.

48 Cao F, Wang S, Cao X, Liu X, Fu K, Hao P, et al. Fibroblast growth factor 21 attenuates calcification of vascular smooth muscle cells in vitro. J Pharm Pharmacol. 2017 Dec;69(12): 1802-16. 